\title{
Transfer and transition practices in 96 European adult congenital heart disease centres
}

\section{Corina Thomet ${ }^{\mathrm{a}, \mathrm{b}, *}$, Markus Schwerzmann ${ }^{\mathrm{a}}$, Werner Budts ${ }^{\mathrm{c}}$, Julie De Backer ${ }^{\mathrm{d}}$, Massimo Chessa ${ }^{\mathrm{e}}$, Gerhard Diller ${ }^{\mathrm{f}}$, Andreas Eicken ${ }^{\mathrm{g}}$, Harald Gabriel ${ }^{\mathrm{h}}$, Pastora Gallego ${ }^{\mathrm{i}}$, Alessandro Giamberti ${ }^{\mathrm{e}}$, Jolien Roos-Hesselink ${ }^{\mathrm{j}}$, Lorna Swan ${ }^{\mathrm{k}}$, Gary Webb ${ }^{1}$, Philip Moons ${ }^{\mathrm{b}, \mathrm{m}, \mathrm{n}}$, on behalf of the ESC Working Group on Grown-up Congenital Heart Disease}

\footnotetext{
a Center of Congenital Heart Disease, Inselspital, Bern University Hospital, University of Bern, Bern, Switzerland

${ }^{\mathrm{b}}$ Department of Public Health and Primary Care, KU Leuven, University of Leuven, Leuven, Belgium

c Congenital and Structural Cardiology, University Hospitals Leuven, Department of Cardiovascular Sciences, KU Leuven, Leuven, Belgium

d Department of Cardiology, Center for Medical Genetics, Ghent University Hospital, Ghent, Belgium

e ACHD Unit - Pediatric and Adult Congenital Heart Centre, IRCCS-Policlinico San Donato, San Donato Milano, Italy

${ }^{\mathrm{f}}$ Division of Adult Congenital and Valvular Heart Disease, Department of Cardiovascular Medicine, University Hospital Muenster, Muenster, Germany

g Division of Pediatric Cardiology and Congenital Heart Disease, German Heart Center Munich, Munich, Germany

${ }^{\text {h }}$ Department of Cardiology, Medical University of Vienna, Vienna, Austria

${ }^{i}$ Adult Congenital Heart Disease Unit, Department of Cardiology, Hospital Universitario Virgen del Rocio, Instituto de BioMedicina de Sevilla (IBIS) and CIBERCV, Sevilla, Spain

j Department of Cardiology, Erasmus Medical Center, Rotterdam, the Netherlands

${ }^{\mathrm{k}}$ Toronto Congenital Cardiac Centre for Adults, Toronto General Hospital, Toronto, Canada

${ }^{1}$ Cincinnati Children's Hospital Heart Institute, Cincinnati, OH, USA

${ }^{\mathrm{m}}$ Institute of Health and Care Science, University of Gothenburg, Gothenburg, Sweden

${ }^{\mathrm{n}}$ Department of Paediatrics and Child Health, University of Cape Town, Cape Town, South Africa
}

\section{A R T I C L E I N F O}

\section{Article history:}

Received 14 September 2020

Accepted 9 November 2020

Available online 1 December 2020

\section{Keywords:}

Heart defects, congenital

Transition to adult care

Quality improvement

Practice guidelines as topic

Europe

\begin{abstract}
A B S T R A C T
Background: Irrespective of initial treatment for congenital heart disease (CHD) in childhood, CHD is a lifelong condition, leaving patients at risk for complications. To support uninterrupted, age- and development-based care for young persons with CHD, guidelines and consensus papers emphasise the need for formal transition programmes, including transfer to adult CHD (ACHD) clinics. Here, we surveyed existing transfer and transition programmes in European ACHD centres. Our aims were to provide a contemporary view of transitional care for patients with $\mathrm{CHD}$ and to evaluate progress over the last decade.

Methods: We conducted a descriptive, cross-sectional survey in 96 ACHD centres in Europe. A specific survey form was developed that sampled the practices of transfer and/or transition. We used a transfer-transition index to quantify adherence to quality indicators of successful transfer and transition.

Results: Of the 96 ACHD centres, 40 (41.7\%) offered a formal transition, and 85 (88.5\%) had structured transfer from paediatric to ACHD care. Although $31 \%$ of the centres performed at a 'good' level on the transfertransition index, only 4 (4.2\%) satisfied all criteria. Most centres with a transition programme offered education and support through a dedicated transition specialist, who was a master's-prepared nurse in most centres. A minority of the ACHD centres offered a flexible transition process, starting at least two years before transfer.

Conclusions: Nearly half of the included ACHD centres offered a formal transition programme, and almost $90 \%$ offered structured transfer. Despite some improvements since 2009, most of the programmes lacked an age- and development-based approach.

(c) 2021 The Authors. Published by Elsevier B.V. This is an open access article under the CC BY-NC-ND license (http://
\end{abstract} creativecommons.org/licenses/by-nc-nd/4.0/).
* Corresponding author at: Center for Congenital Heart Disease, Bern, University Hospital, 3010 Bern, Switzerland.

E-mail address: corina.thomet@insel.ch (C. Thomet).

\section{Introduction}

Congenital heart disease (CHD) is a lifelong condition. Irrespective of initial treatment in childhood, patients are at risk for complications, such as heart failure or arrhythmias. To support patients throughout their entire healthcare life trajectory, lifelong specialised cardiac care is required. For most adults with CHD, follow-up in an expertized centre 
for adult CHD (ACHD) is advised [1,2]. This transfer of care usually takes place when patients are transitioning into adulthood. However, this is not necessarily the case for all centres.

Transition refers to the multi-dimensional process 'that attends to the medical, psychosocial and educational/vocational needs of adolescents as they move from child to adult centred care' [3]. Preparing patients for the journey to independence is an ongoing process that best starts in early adolescence, continues beyond the age of 18 years, and is independent of the complexity of the heart defect $[2,4]$. Hence, paediatric care teams are responsible for initiating this transition. However, collaboration with the ACHD team is certainly beneficial, since the patient will be transferred in most cases to an ACHD centre. As transition is a continuous process, it is advised that transition should not stop after transfer to adult care [5].

Transition programmes have an important role in facilitating uninterrupted, age- and development-based care while patients are transitioning into adulthood [6]. Indeed, the key role of a formal transition process is emphasised by several guidelines and consensus papers [2,5,7-11]. Crucial elements of a smooth transition are (i) initiating the process of transition early, around the age of 12 years, but at least one year before transfer; (ii) having a transition coordinator to facilitate the process; and (iii) providing a written healthcare transition protocol to adolescent patients with CHD and their parents [5,10-12].

About 10 years ago, existing transfer and transition programmes for young people with CHD in Europe and the USA were surveyed [13]. Of the 69 centres included, only one-third offered a formal transition programme [13]. Detailed information from several CHD transition programmes has been published, showing that nurse-led, one-to-one education is the most common intervention implemented [14-17]. This arrangement suggests that specialist nurses play a pivotal role in transitional care for patients with CHD [18].

Over the past few years, several trials have been demonstrated the positive effects of CHD transition programmes [19-22]; more trials are in progress [23-25]. It is, however, unknown to what extent researchbased evidence and transition recommendations have been implemented in daily practice. The overall objective of the present study was to provide a contemporary view of transitional care among patients with CHD. This was accomplished (i) by providing an overview of existing CHD transition programmes in Europe, and (ii) by elaborating on the key quality indicators of transition that should be implemented.

\section{Methods}

We conducted a cross-sectional survey of staff in ACHD centres across Europe. Data collection took place between September 2017 and December 2017. The study was performed according to the principles outlined in the Declaration of Helsinki [26]. However, since the study was considered to be a service quality evaluation, ethical review was not necessary. No patients were surveyed.

\subsection{Study population and procedure}

ACHD centres were eligible for inclusion in the survey if they (i) had on staff at least one cardiologist with ACHD certification or equivalent training, and (ii) had at least 200 ACHD patients in active follow-up at the time of the survey. Overall, 96 specialised European ACHD centres in 24 countries qualified as an ACHD centre according to these criteria, and these contributed survey data. Further information on the study population and procedure of this study have been outlined in a previous paper [27].

\subsection{Measurements and definitions}

For the purpose of the study, we developed a new survey based in part on the survey questionnaire on transfer and transition reported in Hilderson et al. [13]. Our survey questionnaire added structural characteristics and processes of transition that are described in the current literature as key elements of successful transition programmes. It comprised 17 items: 7 questions on transfer and 10 questions on transition. Twelve items required a categorical response, and five items required open-ended questions to be answered. In the present study, we defined structured transfer as 'an event in which adolescents and their parents move their care from the paediatric to the ACHD programme' [28]. A formal transition programme was defined as one 'offering education to support the medical, psychosocial, and educational/vocational needs of adolescents as they move from the child-focused to the adult-focused healthcare system' [3]. Only centres that verified they had a structured transfer or formal transition programme were invited to answer additional questions on key elements of their transfer and transition protocol.

\subsection{Quality indicators of transfer and transition}

On the basis of the most mentioned key elements for transfer and transition that have been described in the literature [2,5,10,12], we constructed a list of quality indicators for the present survey. These indicators were used to classify the centres according to how good or poorly they performed on transfer and transition. For transfer, we identified six quality indicators: (i) having a structured transfer to ACHD care, (ii) transferring all patients to ACHD care, (iii) offering joint or overlapping appointments with someone from the paediatric cardiology team, (iv) offering a flexible transfer period, (v) youth receiving a detailed medical record before leaving paediatric care, and (vi) ACHD team receiving patients' complete medical history from the paediatric care team. For transition, we identified six indicators: (i) having a formal transition programme, (ii) having a dedicated transition specialist, (iii) offering education to the youth, (iv) having a written transition proto$\mathrm{col}$, (v) offering a flexible transition period, and (vi) starting transition for patients $\leq 16$ years old. The performance data of the centres were plotted using a two-dimensional scheme, showing the number of centres per transfer-transition index.

\subsection{Statistical analysis}

Statistical analysis was performed using IBM SPSS Statistics 24 (IBM Corp. Released 2016. IBM SPSS Statistics for Windows, Version 24.0; Armonk, NY: IBM Corp.). Categorical variables are presented as absolute numbers and percentages. Continuous, non-normally distributed variables are presented as medians and interquartile ranges (IQR). To evaluate reliable differences between two independent groups, a Mann-Whitney $U$ Test was calculated. Test statistics with a $p<.05$ were considered statistically significant.

\section{Results}

\subsection{Transfer and transition}

Of the 96 participating ACHD centres, less than half ( $n=40$ centres; $41.7 \%$ ) offered a formal transition programme. However, the majority ( $n=85 ; 88.5 \%$ ) of the centres had a form of structured transfer from a paediatrics provider to an adult-care provider. Of the 40 centres providing transition, 2 did not transfer the patients to ACHD care, yielding 38 ACHD centres (39.6\%) that both had a formal transition programme and had systematically transferred patients to ACHD care (Table 1). Centres having both transfer and transition were mainly concentrated in Belgium, France, the Netherlands, Norway, and the United Kingdom.

Nine centres offered neither structured transfer nor formal transition. These centres were mainly located in Germany, but some also were in Italy, the Netherlands, Spain, and the United Kingdom (Table 1). There was a statistically significant difference between the number of patients seen in centres with transition (median 2000 [IQR: 1235-4491]) and centres without transition (median 1100 [IQR: 
Table 1

Frequency distribution of European centres providing transfer, transition, both, or neither, organised by country.

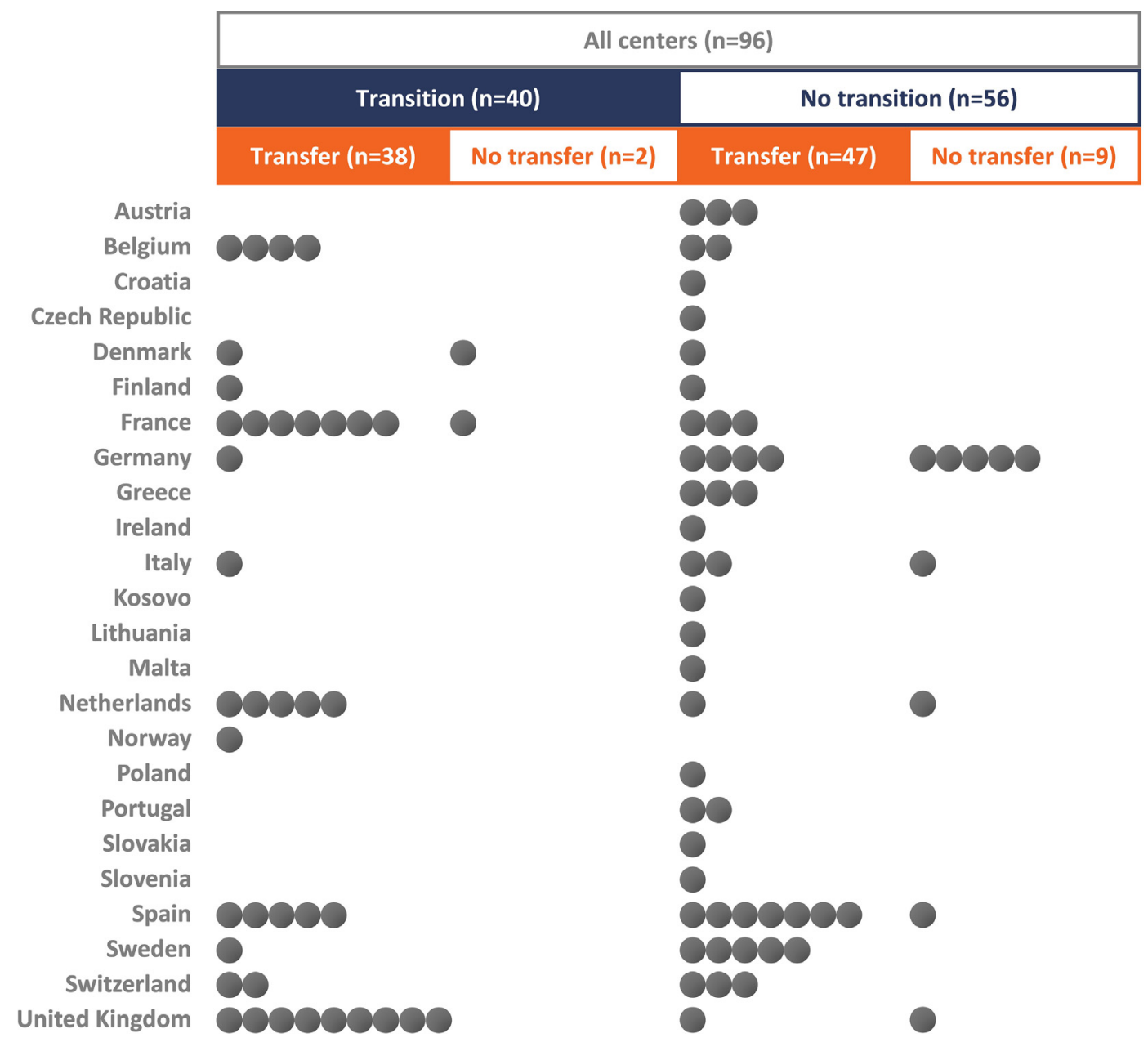

500-2500]); $(\mathrm{U}=723.00, \mathrm{Z}=-2.954, p<.003)$. This was not the case in centres with transfer (median 1500 [IQR: 800-3600]) and centres without transfer (median 1500 [IQR: 654-2200]); (U = 433.00, Z = -0.397, $p<.692)$.

\subsection{Quality indicators for transfer and transition}

\subsubsection{Transfer criteria}

Overall, 85 centres (88.5\%) offered structured transfer. Of all our criteria, the criterion fulfilled by all (100\%) of the centres was 'Receiving a complete medical history from the paediatric care team'. Other criteria were fulfilled less often. For example, 'Offering a flexible transfer to the youth', and 'Offering a detailed medical record to the youth when leaving paediatric care' were fulfilled in $65.1 \%$ and $62.5 \%$ of the centres, respectively (Table 2 ). Centres having both transfer and transition
( $n=33,86.8 \%$ ) offered joint or overlapping appointments with someone from the paediatric team more often than centres having only transfer $(n=25,56.8 \%)$.

\subsubsection{Transition criteria}

Forty centres (41.7\%) had a formal transition programme in place. All these centres had a dedicated transition specialist and offered education to adolescent patients while transitioning to adulthood. The criterion less often fulfilled by the centres was 'Having a transition protocol' (Table 2).

\subsubsection{Transfer-transition index}

The bidimensional performance of the centres in fulfilling the criteria of transfer and transition is depicted in Fig. 1. Only 4 centres (4.2\%) met all quality criteria of transfer and transition (Fig. 1, upper

Table 2

Transfer and transition criteria satisfied by European ACHD centres.

\begin{tabular}{|c|c|c|c|}
\hline Centres meeting transfer criteria, $\mathrm{n}(\%)$ & & Centres meeting transition criteria (\%) & \\
\hline Having a structured transfer to ACHD care & $85(100 \%)$ & Having a formalized transition programme & $40(100 \%)$ \\
\hline Receiving patients complete medical history from paediatric care team & $81(100 \%)$ & Having a dedicated transition specialist & $40(100 \%)$ \\
\hline Offering joint or overlapping appointments with someone from the paediatric cardiology team & $58(70.7 \%)$ & Offering education to the youth & $40(100 \%)$ \\
\hline Transferring all patients to ACHD care & $55(69.6 \%)$ & Starting transition at an age $\leq 16$ years & $32(80 \%)$ \\
\hline Offering a flexible transfer period & $54(65.1 \%)$ & Offering a flexible transition period (start or end) & $28(70 \%)$ \\
\hline Medical record from paediatric care team given to the youth & $50(62.5 \%)$ & Having a written transition protocol & $24(61.5 \%)$ \\
\hline
\end{tabular}




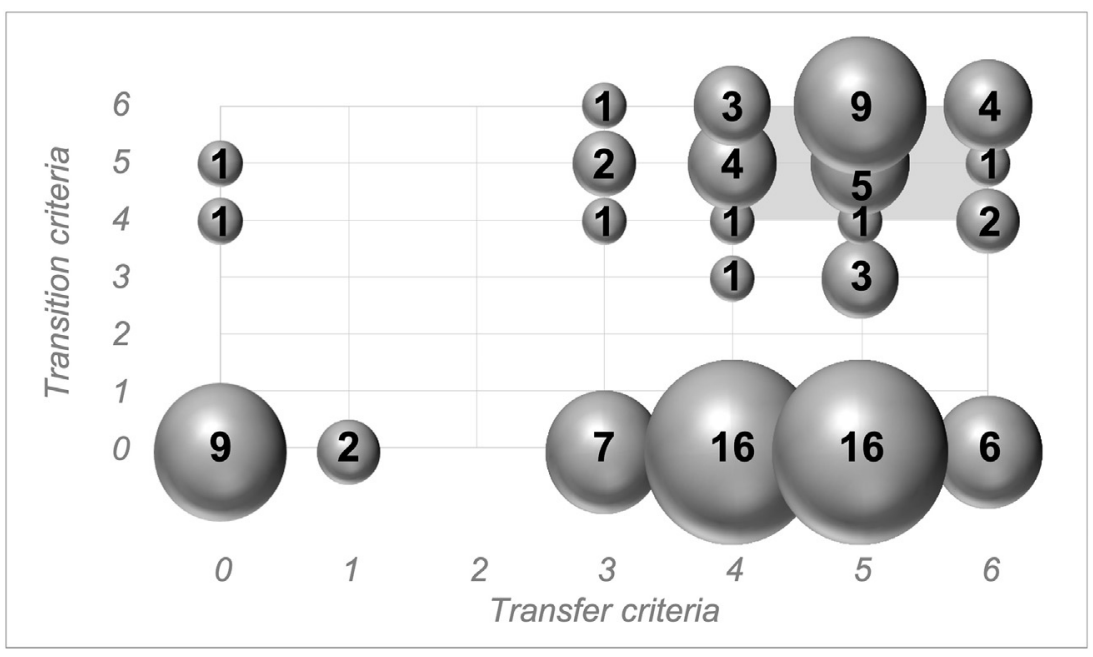

Fig. 1. Bubble graph showing the number of centres per transfer-transition index.

right corner of graph). The overall performance of centres could be considered to be 'good' if they met at least four criteria on both the transfer and transition dimension, as indicated by the grey zone in Fig. 1. Thirty centres (31\%) achieved this level of performance. A large proportion of the centres fulfilled three or more transfer criteria but failed to meet any transition criterion.

\subsection{Transition: Personnel and educational aspects}

Generally, more non-medical staff were working in centres with transition (83.1\% vs. $40.6 \%)$. Centres with transition had a median of 2 (IQR: 1-3) dedicated transition specialists, with a median full-time equivalent of 50\%. Master's-prepared nurses (median 1 [IQR: 1-3]); physicians (median 1 [IQR: 0.5-1]); and psychologists (median 0 [IQR: $0-0$ ] were working as transition specialists. Five centres used alternative kinds of personnel for staffing, such as dietitians, nurse specialists, peer counsellors, or staff from local patient charity and patient associations. Of the 40 centres with transition, 23 (57.5\%) had a multidisciplinary transition team consisting of master's-prepared nurses in combination with physicians, psychologists, or social workers.

Centres also offered educational opportunities, but differed in kinds and number. Regarding transition interventions, 27 of the centres (67.5\%) offered one-to-one counselling, and 12 (30\%) offered group sessions and one-to-one counselling. Moreover, 7 centres offered other additional interventions, such as youth camps, opening events, annual young adult clinics, or patient days. Only 1 of the 7 centres $(2.5 \%)$ had no additional one-to-one or group consultations. On average, a transition consultation lasted $45 \mathrm{~min}$ (IQR 30-60 min).

\subsection{Transfer and transition: Time frame}

Of the 85 centres with transfer, 29 transferred their patients when the patients reached a fixed pre-set age, which differed across centres. For these centres, patients were transferred at a median age of 18 years (IQR: 17-18). For centres using a flexible age range for transfer decisions, transfer occurred between the ages of 16 years (IQR: 15-16) and 19 years (IQR: 18-20).

Regarding the transition process, of the 40 centres, 10 (25\%) started their transition at a pre-set age (median 15 years, IQR: 12.8-18), while 11 centres (28.2\%) completed their transition at a predefined age (median $18 \mathrm{y}$, IQR: 18-19). For the other centres using a flexible age range, $54(65.1 \%)$ transferred at a flexible age range, $28(70 \%)$ offered a flexible transition start, and 24 (61.5\%) had a flexible transition completion. Fig. 2 depicts the great range we observed for the transfer and transition time frame. First, for 19 centres (50\%), transfer to adult care and the first transition consultation started simultaneously. Second, for $23(60.5 \%)$ centres, the transition process ended immediately after transfer to adult care. Preferably, the transition process should start at least two years before the transfer and only should end after transfer to ACHD care, with termination based on the patient's individual developmental needs. This pattern was observed for centre nos. 4, 6, 24, and 35 (Fig. 2).

\section{Discussion}

The implementation of a formal transition programme for patients with CHD, a scheme that addresses transfer to adult care for adolescent patients and that includes their families, has been advised by numerous consensus, position, and guideline papers [2,5,9]. However, these guidelines are followed to varying degrees. Transfer and transition programmes are critical tools in providing the kind of life-long care $\mathrm{CHD}$ patients need. Through the use of a quantitative survey, the present study provided an overview of the current numbers of ACHD centres in Europe that offer transfer and transition. In addition, it evaluated established key criteria in their ability to detect to what extent recommendations have been implemented in practice.

Our results relate to those of a previous study by Hilderson et al. published in 2009 on the practice of transfer and transition in 69 CHD hospitals in Europe and the USA [13]. Comparing our results with that study indicates that over the past 10 years, the recommendation for implementing formal transfer and transition programmes has increasingly been adopted [13]. A top-level comparison of the two complete sets of data shows that transfer programmes have increased overall by over $15 \%$ and transition programmes by over $13 \%$. However, when we compare the European subset of data from the Hilderson et al. study ( $n=26$ centres $^{1}$ ) with the present study, the outcome is more sobering. The next sections present this comparison of European centres in detail.

\subsection{Transfer and transition practice}

The vast majority of the 96 European ACHD centres surveyed in the present study offered structured transfer to adult care. This is an increase of $7.2 \%$ ( $88.5 \%$ vs. $81.3 \%$ ) compared to the data of the 26 European centres sampled in the 2009 study [13]. The percentage of centres with formal transition programmes, however, decreased by

\footnotetext{
${ }^{1}$ Data from the Hilderson et al. study (2009) was made available by the authors so that we could directly compare results from that study with the present study.
} 

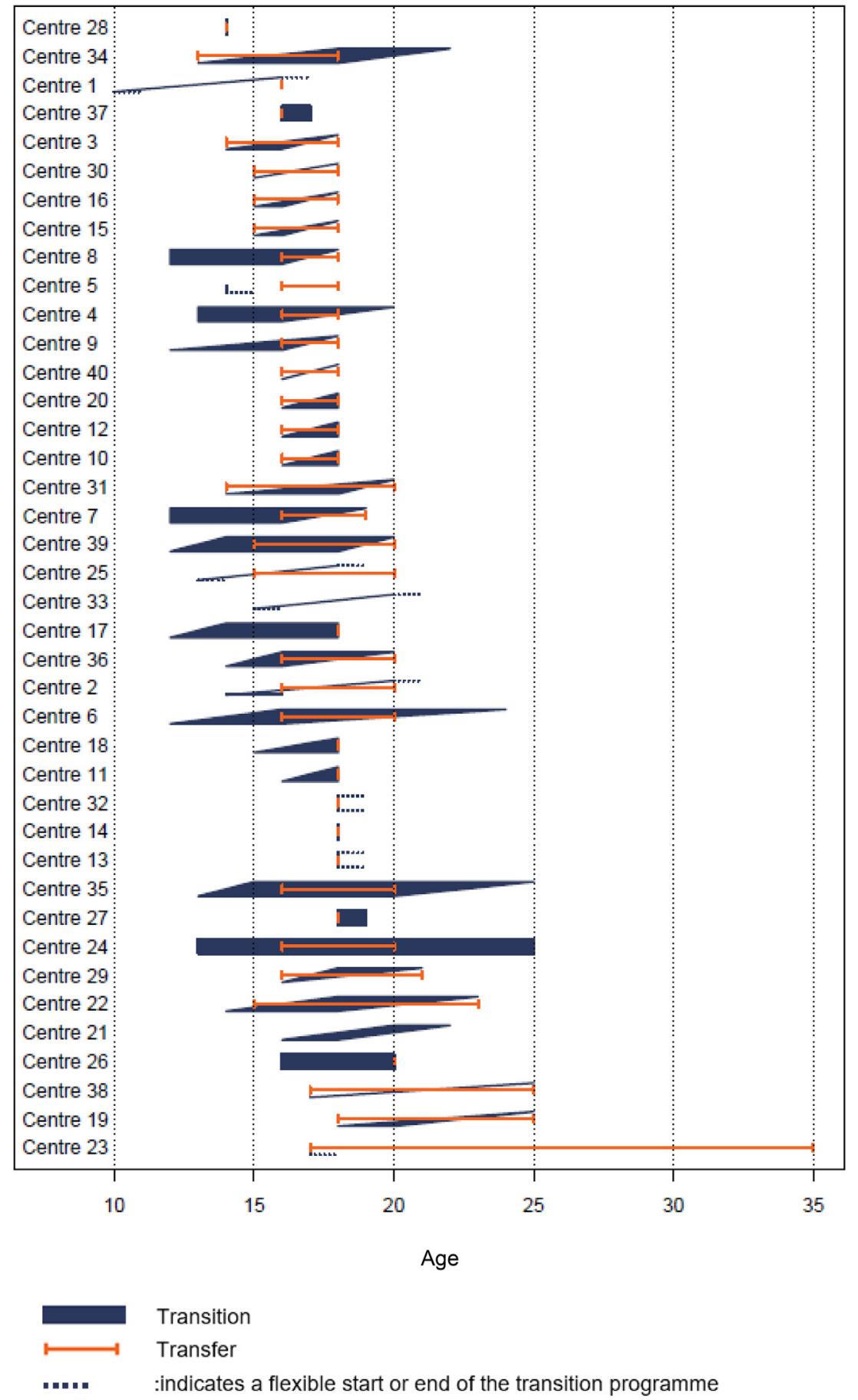

Fig. 2. Range of observed transfer and transition periods for ACHD centres that offered a formal transition programme, stratified by centre ( $n=40$ ).

$8.3 \%$ ( $41.7 \%$ vs. $50 \%$ ). The majority of centres offering transition in our study also indicated that they have structured transfer. Only a minority (10\%) offered neither transfer nor transition.

The data imply that over time, European ACHD centres invested merely in structured transfer procedures and less in the development of transition programmes. Our results showed further that ACHD centres that treat more patients also have an established transition programme significantly more often than centres that treat fewer patients. This suggests that it might be more difficult for small centres to establish a formal transition programme.

\subsection{Transfer and transition criteria}

Our quantitation of transfer and transition practices criteria using the transfer-transition index revealed some interesting practices of European ACHD centres. Almost 30\% of the centres performed well on satisfying the criteria (i.e., met at least four transfer and transition criteria). Four centres achieved the highest possible score on the transfer-transition index, indicating they satisfied all transfer and transition criteria. The specific transfer criterion, 'receiving a complete medical record from the paediatric team at the time of transfer' was satisfied 
by all centres that had structured transfer. Transfer criteria that were less often met were mostly related to collaboration issues with the paediatric team as well as to time resources. These criteria include (i) offering joint or overlapping appointments, (ii) transferring all patients, (iii) offering a flexible transfer period, and (iv) offering medical records for youths. Whether centres were able to offer flexible transfer based on the patients' developmental maturity often depended on regulations of the centre's country where it was located or the institution's requirements. For example, in Sweden, it is mandatory to transfer patients by the age of 18 years. This is not necessarily problematic if a structured transition programme includes both paediatric and ACHD care teams. On the other hand, a flexible transfer policy may meet individual patient's needs better, since it ensures that transfer of care can take place at a time when the patient is medically stable.

Remarkably, one-third of the centres surveyed in the present study do not transfer all patients to ACHD care at some point. This proportion has increased by $15 \%$ since the Hilderson et al. study in 2009 , which reported that only $15.4 \%$ of centres fail to do this transfer at some point. Hence, the situation has deteriorated in the past decade, despite the suggestion in the latest recommendations that all CHD patients should be seen at least once by an ACHD cardiologist [1]. This is critical, because a cardiologist specialising in adult CHD care is in the best position to give advice on the level of adult care needed [1].

Since the transition process is multi-dimensional and includes educational/vocational aspects besides medical and psychosocial ones [3], our survey also sampled performance of centres on educational programmes. The specific transition criteria for this aspect was, 'Offering education to the youth' and 'Having a dedicated transition specialist'. All centres with a formal transition programme reported offering specialised education to patients and also said they had a dedicated transition specialist on staff.

Master's-prepared nurses play an important role in providing these services by offering one-to-one counselling or team-counselling with another healthcare provider. Different authors have underscored the important role of master's-prepared nurses in the transition process $[29,30]$. They can be pivotal in the role of transition coordinator $[21,25,31,32]$. In line with our results, examples of transition programmes in the literature showed that such nurses in this role as transition coordinator offer individualised and structured support, guidance, and education to patients and parents [14-16,33]. Our survey also showed that some transition criteria regarding this aspect were met less frequently. These include criteria that require more flexibility from staff, such as starting transition before the age of 16 or offering a flexible start or end of the transition process. Criteria that imply collaboration between hospitals were also met less frequently.

\subsection{Benchmarking and establishment of a transition programme}

Transfer and transition criteria are important indicators not only for benchmarking, or standardising, but also for establishing a formal transition programme. It has been reported that for adolescents with chronic conditions, transition programmes more often emphasise medical aspects at the expense of neglecting individual, adolescent-related aspects [34]. Our results on practices of ACHD centres in Europe are in line with those observations. For example, only 4 centres (10\%) offered a transition process that was clearly based on patients' needs, specifically one that starts at least two years before the transfer and only ends after the transfer to ACHD care. This result is surprising, as it has been reported that such an individual-needs approach is strongly advocated because it takes into account individual differences in biological growth. Moreover, it tempers the impact of social-role transitions that adolescent patients navigate while transitioning into adulthood [35].

Altogether, the number of ACHD centres offering formal transition has grown in Europe over the past 10 years. However, only the minority of centres follow the recommendations on transfer and transition to the fullest possible extent. Therefore, questions arise: Are the recommendations-often expert-based-overstated or just too difficult to fulfil for most centres due to financial and/or organisational restrictions? To allow reasonable benchmarking, further insights are needed into key indicators of how centres offer transfer and transition, and how this is linked to outcome.

\section{Limitations}

The survey data summarised in this report reflect the status of transfer and transition situations of a large number of European ACHD centres in 2016. Due to continuously evolving practices in the field, however, the situation might be somewhat different in 2020, and centres may have improved. In addition, even though the survey was conducted very broadly using the networks of multiple international and national organisations and providers, we cannot assume that we identified every specialised ACHD centre in Europe that has formal transition and transfer. However, with almost 100 centres surveyed, we are confident that we have provided a reasonable contemporary view of transfer and transition of CHD patients in Europe. It is important to state that the data are self-reported data, and verification was not possible. To achieve the highest possible comparability, participants were provided with specific clear definitions of the terms transfer and transition. Also, the benefits of structural transfer and formal transition could not be investigated, since quality outcomes are not well established in this field yet. In addition, we were not able to determine whether more patients had been transferred to an ACHD centre if paediatric cardiology and the ACHD centre were located at the same hospital, or if patients needed to transfer to another healthcare system for ACHD care; our main study aim was to determine how many ACHD centres exist in Europe. Our study did not include questions about transfer legislation. Since a country's legislation mandates at what age transfer occurs, this limitation needs to be considered when interpreting the data.

\section{Conclusion}

It is widely recognised that it is crucial to support and educate adolescent patients with CHD to take charge of their life and health. This is largely accomplished by engagement in a formal transition programme, including transfer to adult care. In recent years, it is clear that nearly half of the ACHD centres in Europe have implemented at least transition elements in their clinics, and almost 90\% offer structured transfer from paediatric to adult care. Despite this tremendous achievement, improvements involving developmental-related needs of young patients have to be met. Furthermore, it is important to determine why one-third of patients still are not transferred to ACHD care and how recommended transfer and transition criteria are linked to outcomes.

\section{Financial disclosure}

None.

\section{Declaration of Competing Interest}

The authors report no relationships that could be construed as a conflict of interest.

\section{Acknowledgments}

This work was supported by the ESC Working Group on Grown-Up Congenital Heart Disease www.escardio.org/Working-groups/WorkingGroup-on-Adult-Congenital-Heart-Disease/About We thank all participating centres who contributed to this study. 


\section{References}

[1] H. Baumgartner, W. Budts, M. Chessa, et al., Recommendations for organization of care for adults with congenital heart disease and for training in the subspecialty of 'Grown-up congenital heart Disease' in Europe: a position paper of the working group on grown-up congenital heart disease of the European Society of Cardiology, Eur. Heart J. 35 (2014) 686-690.

[2] K.K. Stout, C.J. Daniels, J.A. Aboulhosn, et al., 2018 AHA/ACC guideline for the Management of Adults with Congenital Heart Disease: a report of the American College of Cardiology/American Heart Association task force on clinical practice guidelines, J. Am. Coll. Cardiol. 73 (2019) e81-e192.

[3] R.W. Blum, D. Garell, C.H. Hodgman, et al., Transition from child-centered to adult health-care systems for adolescents with chronic conditions. A position paper of the Society for Adolescent Medicine, J. Adolesc. Health 14 (1993) 570-576.

[4] Å. Burström, M. Acuña Mora, M. Öjmyr-Joelsson, et al., Ready for transfer to adult care? A triadic evaluation of transition readiness in adolescents with congenital heart disease and their parents, J. Fam. Nurs. 25 (2019) 447-468.

[5] C. Sable, E. Foster, K. Uzark, et al., Best practices in managing transition to adulthood for adolescents with congenital heart disease: the transition process and medical and psychosocial issues: a scientific statement from the American Heart Association, Circulation. 123 (2011) 1454-1485.

[6] P. Moons, D. Hilderson, K. Van Deyk, Implementation of transition programs can prevent another lost generation of patients with congenital heart disease, Eur. J. Cardiovasc. Nurs. 7 (2008) 259-263.

[7] E. Foster, T.P. Graham Jr., D.J. Driscoll, et al., Task force 2: special health care needs of adults with congenital heart disease, J. Am. Coll. Cardiol. 37 (2001) 1176-1183.

[8] American Academy of Pediatrics, American Academy of Family Physicians, American College of Physicians, Transitions Clinical Report Authoring Group, W.C. Cooley, P.J. Sagerman, Supporting the health care transition from adolescence to adulthood in the medical home, Pediatrics 128 (2011) 182-200.

[9] American Academy of Pediatrics, American Academy of Family Physicians, American College of Physicians-American Society of Internal Medicine, A consensus statement on health care transitions for young adults with special health care needs, Pediatrics 110 (2002) 1304-1306.

[10] J. Deanfield, E. Thaulow, C. Warnes, et al., Management of grown up congenital heart disease, Eur. Heart J. 24 (2003) 1035-1084.

[11] C.A. Warnes, R.G. Williams, T.M. Bashore, et al., ACC/AHA 2008 guidelines for the management of adults with congenital heart disease: a report of the American College of Cardiology/American Heart Association Task Force on Practice Guidelines (Writing Committee to Develop Guidelines on the Management of Adults With Congenital Heart Disease). Developed in Collaboration With the American Society of Echocardiography, Heart Rhythm Society, International Society for Adult Congenital Heart Disease, Society for Cardiovascular Angiography and Interventions, and Society of Thoracic Surgeons, J. Am. Coll. Cardiol. 52 (2008) e143-e263.

[12] J.C. Suris, C. Akre, Key elements for, and indicators of, a successful transition: an international Delphi study, J. Adolesc. Health 56 (2015) 612-618.

[13] D. Hilderson, A.S. Saidi, K. Van Deyk, et al., Attitude toward and current practice of transfer and transition of adolescents with congenital heart disease in the United States of America and Europe, Pediatr. Cardiol. 30 (2009) 786-793.

[14] S.K. Berg, P.G. Hertz, Outpatient nursing clinic for congenital heart disease patients: Copenhagen transition program, J. Cardiovasc. Nurs. 22 (2007) 488-492.

[15] C. Thomet, C. Lindenberg, M. Schwerzmann, E. Spichiger, Adolescents' with congenital heart disease and their parents' experiences of a nurse-led transition program. An interpretive phenomenological study, Pflege. 31 (2018) 9-18.

[16] M. de Hosson, J. De Backer, D. De Wolf, et al., Development of a transition program for adolescents with congenital heart disease, Eur. J. Pediatr. 179 (2020) 339-348.
[17] M. Ladouceur, J. Calderon, M. Traore, et al., Educational needs of adolescents with congenital heart disease: impact of a transition intervention programme, Arch. Cardiovasc. Dis. 110 (2017) 317-324

[18] P. Moons, W. Scholte Op Reimer, S. De Geest, et al., Nurse specialists in adult congenital heart disease: the current status in Europe, Eur. J. Cardiovasc. Nurs. 5 (2006) 60-67.

[19] A.S. Mackie, G.R. Rempel, A.H. Kovacs, et al., Transition intervention for adolescents with congenital heart disease, J. Am. Coll. Cardiol. 71 (2018) 1768-1777.

[20] A.S. Mackie, S. Islam, J. Magill-Evans, et al., Healthcare transition for youth with heart disease: a clinical trial, Heart. 100 (2014) 1113-1118.

[21] S.F. Flocco, F. Dellafiore, R. Caruso, et al., Improving health perception through a transition care model for adolescents with congenital heart disease, J. Cardiovasc. Med. (Hagerstown) 20 (2019) 253-260

[22] A.C. Hergenroeder, D.S. Moodie, D.J. Penny, et al., Functional classification of heart failure before and after implementing a healthcare transition program for youth and young adults transferring from a pediatric to an adult congenital heart disease clinics, Congenit. Heart Dis. 13 (2018) 548-553.

[23] M. Saarijarvi, L. Wallin, P. Moons, H. Gyllensten, E.L. Bratt, Transition program for adolescents with congenital heart disease in transition to adulthood: protocol for a mixed-method process evaluation study (the STEPSTONES project), BMJ Open 9 (2019), e028229,

[24] M. Acuna Mora, M. Saarijarvi, C. Sparud-Lundin, P. Moons, E.L. Bratt, Empowering young persons with congenital heart disease: using intervention mapping to develop a transition program - the STEPSTONES project, J. Pediatr. Nurs. 50 (2020) e8-e17.

[25] M. Acuna Mora, C. Sparud-Lundin, E.L. Bratt, P. Moons, Person-centred transition programme to empower adolescents with congenital heart disease in the transition to adulthood: a study protocol for a hybrid randomised controlled trial (STEPSTONES project), BMJ Open 7 (2017), e014593,

[26] World Medical Association, World Medical Association Declaration of Helsinki. Ethical Principles for Medical Research Involving Human Subjects, 79, Bulletin of the World Health Organization, 2001 373-374.

[27] C. Thomet, P. Moons, W. Budts, et al., Staffing, activities, and infrastructure in 96 specialised adult congenital heart disease clinics in Europe, Int. J. Cardiol. 292 (2019) 100-105.

[28] A. Knauth Meadows, V. Bosco, E. Tong S. Fernandes, A. Saidi, Transition and transfer from pediatric to adult care of young adults with complex congenital heart disease, Curr. Cardiol. Rep. 11 (2009) 291-297.

[29] P. Moons, S. De Geest, W. Budts, Comprehensive care for adults with congenital heart disease: expanding roles for nurses, Eur. J. Cardiovasc. Nurs. 1 (2002) 23-28.

[30] C.L. Betz, G. Redcay, Dimensions of the transition service coordinator role, J. Spec. Pediatr. Nurs. 10 (2005) 49-59.

[31] D. Hilderson, R. Westhovens, C. Wouters, K Van der Elst, E Goossens, P. Moons, Rationale, design and baseline data of a mixed methods study examining the clinical impact of a brief transition programme for young people with juvenile idiopathic arthritis: the DON'T RETARD project, BMJ Open 3 (2013), e003591,

[32] A.L. Brorsson, E.L. Bratt, P. Moons, et al., Randomised controlled trial of a personcentred transition programme for adolescents with type 1 diabetes (STEPSTONESDIAB): a study protocol, BMJ Open 10 (2020), e036496,

[33] H. Habibi, Y. Emmanuel, N. Chung, Process of transition for congenital heart patients: preventing loss to follow-up, Clin. Nurse Specialist CNS. 31 (2017) 329-334.

[34] J.N.T. Sattoe, S.R. Hilberink, A. van Staa, How to define successful transition? An exploration of consensus indicators and outcomes in young adults with chronic conditions, Child Care Health Dev. 43 (2017) 768-773.

[35] S.M. Sawyer, P.S. Azzopardi, D. Wickremarathne, G.C. Patton, The age of adolescence, Lancet Child Adolesc. Health. 2 (2018) 223-228 